Exp Brain Res (1988) 72: 117-128

\title{
Time constants of facilitation and depression in Renshaw cell responses to random stimulation of motor axons
}

\author{
Y. Laouris, U. Windhorst, R. Rissing, U. Kuipers, and J. Meyer-Lohmann \\ Zentrum Physiologie und Pathophysiologie der Universität, Abteilung Neuro- und Sinnesphysiologie, Humboldtallee 23, \\ D-3400 Göttingen, Federal Republic of Germany
}

Summary. In 9 adult anaesthetized cats, 22 lumbosacral Renshaw cells recorded with $\mathrm{NaCl}$-filled micropipettes were activated by random stimulation of ventral roots or peripheral nerves. The stimulus patterns had mean rates of $9.5-13$ or $20-23$ or 45 pulses per second and were pseudo-Poisson; short intervals below ca. $5 \mathrm{~ms}$ (except in two cases) were excluded. The Renshaw cell responses were evaluated by two kinds of peristimulus-time histograms (PSTHs). "Conventional" PSTHs were calculated by averaging the Renshaw cell discharge with respect to all the stimuli in a train. These PSTHs showed an early excitatory response which was often followed by a longer-lasting slight reduction of the discharge probability. These two response components were positively correlated. "Conditional" PSTHs were determined by averaging the Renshaw cell discharge with respect to the second ("test") stimulus in pairs of stimuli which were separated by varied intervals, $\delta$. The direct effect of the first "conditional" response was subtracted from the excitation following the second ("test") stimulus so as to isolate the effect caused by the second stimulus per se. After such a correction, the effect of the first "conditioning" stimulus showed pure depression, pure facilitation or mixed facilitation/depression. Analysis of such conditioning curves yielded two time constants of facilitation (ranges: ca. 4-35 ms and 93-102 ms) and two of depression (ranges: ca. 7-25 ms and 50-161 ms). It is concluded that these time constants are compatible with processes of short-term synaptic plasticity known from other synapses. Other processes such as afterhyperpolarization and mutual inhibition probably are of less importance.

Key words: Renshaw cells - Motor axons -Random stimulation - Synaptic transmission - Facilitation Depression - Time constants

Offprint requests to: $\mathrm{U}$. Windhorst (address see above)

\section{Introduction}

Renshaw cells have been implicated in the finestructuring of motoneuronal discharge (reviewed by Windhorst 1988). Under natural conditions, the input to Renshaw cells is not constant. Even the "steady-state" discharge of a single motoneurone, which may strongly excite a Renshaw cell (Ross et al. 1975; van Keulen 1981; see also Hamm et al. 1987), is not completely regular, but fluctuates more or less around a mean rate. To understand the contribution of Renshaw cells to motoneurone firing fluctuation, a precise characterization of their dynamic behaviour is necessary. In an attempt to quantitatively describe the dynamic signal transmission between motor axons and Renshaw cells, we have recorded the latter's discharges to random stimulation of motor axons. This input pattern has a number of advantages, among which computational economy and the possibility to perform spectral computations and nonlinear analysis on the same data stand out (see Methods). Spectral analysis, used to compute the frequency response, revealed that the coherence deviated from a value of 1 (corresponding to linear signal transmission in the absence of noise interference), depending on the input parameters such as strength of excitatory coupling and mean stimulus rate (Christakos et al. 1987). To determine the possible contribution of nonlinear system properties to this deviation, a particular nonlinear analysis has been added (Windhorst et al. 1987). It appeared that the Renshaw cell responses to the random stimuli depended on the previous history of activation in that they could be facilitated by stimuli presented just before and/or depressed by stimuli occurring at intervals up to several hundred milliseconds earlier. This pattern of conditioning was very similar to that found at neuromuscular and various central synapses. To support this analogy, a precise determination of the time course of the processes would be 
helpful. A comparison of this time course with that at other synapses, particularly at neuromuscular junctions (which are from the same neurone and thus also operated by acetylcholine), would allow an inference by analogy as to the mechanisms responsible for the decay processes at central synapses that are not readily accessible to experimental manipulation. The aim of the present study is, firstly, to contribute to the precise description of Renshaw cell behaviour by analysis of some nonlinear features and, secondly, to provide clues to the possible origin of the observed nonlinear effects.

\section{Methods}

\section{Experimental techniques}

The experiments were performed on 9 adult cats of either sex weighing between 3.3 and $5.0 \mathrm{~kg}$, and anaesthetised with pentobarbitone (initially $40 \mathrm{mg} / \mathrm{kg}$ i.p., supplemented by smaller intravenous doses), or with urethane-chloralose $(400 / 40 \mathrm{mg} / \mathrm{kg}$ i.p.). Seven cats were spinalized between the $L_{1}$ and $L_{2}$ vertebrae. Tracheal, arterial (A. carotis comm.) and venous (V. jugularis ext.) canulae were inserted for artificial respiration after curarization (Pancuronium ${ }^{\circledR}$ ), blood pressure monitoring and application of drugs or infusions. Blood pressure was kept above $80 \mathrm{~mm} \mathrm{Hg}$ by infusion of dectrane and/or Ringer solution when necessary, which was rarely the case, however. End-tidal $\mathrm{CO}_{2}$ was measured and usually lay between 3.5 and $6 \mathrm{Vol} \%$. Rectal temperature and that of the spinal paraffine pool were kept between $37-38$ and $35-37^{\circ} \mathrm{C}$, respectively, by radiant heat.
After a laminectomy in the lumbar region, dorsal roots $\mathrm{L}_{6}$ to $S_{2}$ were cut on the experimental side (left). The intact ventral roots $\mathrm{L}_{\mathcal{7}}$ and $\mathrm{S}_{1}$ were put on bipolar platinum electrodes for stimulation or recording. A number of peripheral nerves were prepared for stimulation: the "hamstring" nerve which in later experiments was split into several constituent nerve branches (anterior and posterior biceps femoris, anterior and posterior semitendinosus; semimembranosus); the gastrocnemius nerves which in some cases were split into several naturally occurring nerve branches; the "tibial" nerve (without gastrocnemii); and the common peroneal nerve. Ventral roots or nerves were stimulated applying rectangular voltage pulses of $0.05-0.1 \mathrm{~ms}$ width.

Activity of 22 lumbar Renshaw cells (in $\mathrm{L}_{7}$ or $\mathrm{S}_{1}$ segments) was recorded extracellularly with micropipettes filled with 3-molar $\mathrm{NaCl}$ solution. They were identified by their responses to low-rate (2-3 pulses per second: pps) regular stimulation of a ventral root, the responses exhibiting a small latency $(0.5-1.2 \mathrm{~ms})$, and a burstlike (up to 50-60 ms length), high-rate discharge (up to $1500 \mathrm{pps}$ ).

\section{Stimulus patterns, data processing and evaluation}

During the test periods, ventral roots or nerves or nerve branches to various muscles were stimulated with random sequences of brief electrical rectangular pulses, at various strengths. Three different ranges of mean stimulus rate were employed because the extent and duration of the conditioning effects observed (early facilitation and/or subsequent depression which could last some hundreds of milliseconds) depended on the mean stimulus rate (Čapek and Esplin 1977; Windhorst et al. 1987). The mean stimulus rate of the random sequences we used lay between 9.5 and 13 (low: "l"), between 20 and 23 (medium: "m") and, in one case, near 45 (high: "h") pulses per second (pps). These mean rates were chosen to lie within the range of natural steady firing rates of $\alpha$-motoneurones.

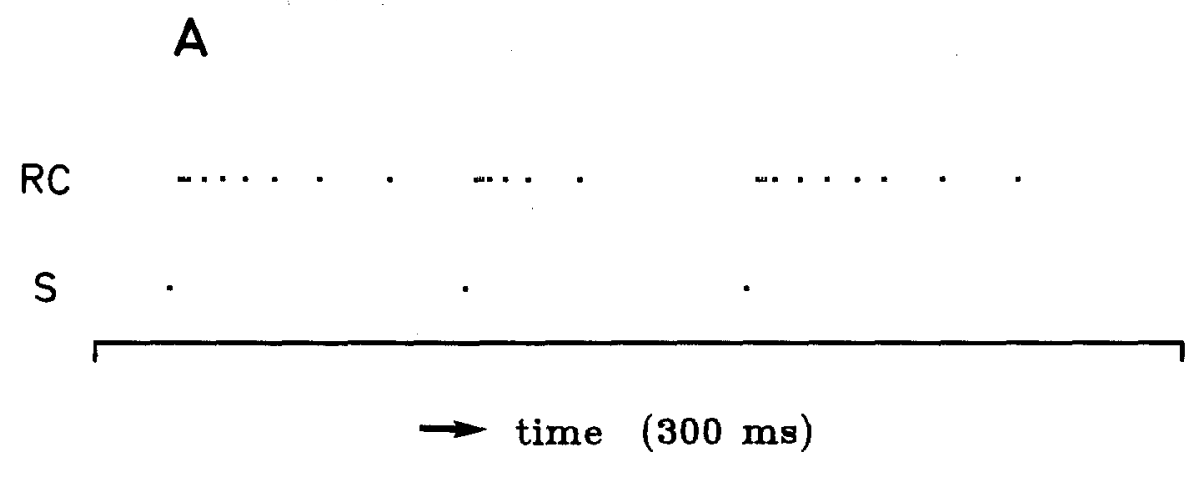

B

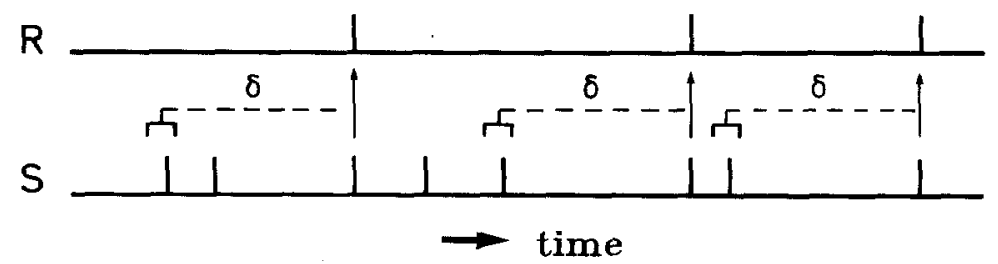

Fig. 1A, B. Example of sampled data (A) and scheme of the selection of stimulus pairs from the stimulus train (B). A Shows a 300-ms section of sampled data containing the response of a Renshaw cell (upper trace labelled RC) to random stimulation (lower trace labelled S) of the medial gastrocnemius nerve at $400 \mathrm{mV}$ and a mean rate of $12.5 \mathrm{pps}$. B Illustrates the procedure of selecting, as reference events, pairs of stimuli, which were separated by a defined (average) interval $\delta$. From the original stimulus train (labelled S) were selected and written on a reference channel (labelled $\mathrm{R}$ ) those stimuli that were preceded by other stimuli within a time window of $5 \mathrm{~ms}$ width, located on average $\delta \mathrm{ms}$ before 
To avoid refractoriness of the motor axons, short interstimulus intervals below about $5 \mathrm{~ms}$ were excluded from the stimulus sequences. These stimulus patterns thus corresponded to "pseudo-Poisson" patterns, i.e., had exponential interval distributions and flat auto-correlograms except that short intervals below $5 \mathrm{~ms}$ were absent. Each stimulation run usually lasted several (more than three up to 15 or 20) min. Since most cells were tested with various input constellations and stimulus parameters, their testing lasted between some minutes and several (4-5) hours.

The pertinent data were stored on analogue tape before computer sampling for off-line analysis (PDP 11/23). Stimulus and Renshaw cell spike trains were sampled at a rate of $10 \mathrm{kHz}$, that is, with an accuracy of $0.1 \mathrm{~ms}$. An example of computer-sampled data is shown in Fig. 1A, which presents a $300-\mathrm{ms}$ dot display of a Renshaw cell's activity (upper trace labelled RC) to three stimuli (labelled S) delivered to the medial gastrocnemius nerve at a mean rate of $12.5 \mathrm{pps}$. Time-domain analysis was performed by computing peri-stimulus time histograms (PSTHs) of two types. (a) The first type was the "conventional" PSTH, computed with respect to all the successive stimuli occurring in one experimental run. (b) The second type corresponded to "conditional" PSTHs, which were calculated with respect to pairs of stimuli (see Fig. 1B). From the original stimulus train (labelled S) those stimuli that were preceded by other stimuli within a time window of $5 \mathrm{~ms}$ width, located on average $\delta \mathrm{ms}$ before were selected and written on a reference channel (labelled R). Renshaw cell discharges were then correlated with respect to these conditioned stimuli yielding "conditional" PSTHs. Note that the selected pairs of stimuli are compound events which occur randomly as do the original stimuli. Pseudo-Poisson inputs are indeed optimal for this kind of analysis because their autocorrelation is flat for $\delta$ greater than the minimal interval. The stimulus pairs defined for different $\delta$ 's $(\geq 7.5 \mathrm{~ms})$ therefore have equal probability of occurrence. The preceding stimuli in the pairs can be envisaged as "conditioning" and the following ones as "test" stimuli. With respect to these pairs of stimuli, all the others, including those falling between the paired stimuli, occur randomly and thereby contribute only to the mean rate of Renshaw cell firing.

The advantages of the random stimulus patterns used may be summarized as follows. (1) This type of input is very suitable for both linear and nonlinear analysis, i.e., the same experimental data can be subjected to both sorts of analysis, which is highly desirable for comparison. Linear analysis involves the computation of frequency responses, including estimates of coherence, gain and phase functions, and has been widely used in the quantitative characterization of physiological systems (for references see Christakos et al. 1987). Nonlinear analysis as presented in this paper is based on a well-established method (white-noise analysis; see Windhorst et al. 1983) and has been employed previously by a number of workers. (2) The use of random inputs is very economical. For example, to obtain equivalent results with sinusoidal inputs (in linear analysis) or with paired stimuli repeated at low rate (as often used in studies of synaptic facilitation and depression) takes much more time and thus poses higher demands on the stability of the preparation and recording. (3) The mean rate of the pulse trains is of great importance for the conditioning effects to be described. To study these effects with the classical double-pulse approach at mean rates corresponding to steady motoneurone firing rates would restrict the analysis to very brief intervals between the double pulses. Regular pulse trains (as used by Haase 1963, for example) would not yield the same results as random inputs, whose most characteristic feature is the moment-to-moment fluctuation of instantaneous rates around a mean, which is the feature under study in this approach. Indeed one of the prominent advantages of random stimulus patterns is that they contain a wide range of interpulse intervals within even short time spans.

\section{Results}

More than 50 experimental runs with different ventral roots, nerves and stimulus parameters were performed on 22 Renshaw cells. From these, 26 runs from 11 cells were selected for the determination of time constants because their conditioning curves were smooth enough.

\section{Long-lasting conditioning of Renshaw cell responses: $\delta$-curves}

The Renshaw cell responses to random sequences of stimuli to motor axons consisted of single impulses or small bursts of discharges, the magnitude depending on the mean stimulus rate, on the strength of synaptic coupling of the axons to the cell and on the pervious history of the stimulus sequence (Windhorst et al. 1987, and unpublished results; see also below). The average response to each stimulus was assessed by computing a "conventional" PSTH. This is exemplified in Fig. 2a (lower right PSTH) for the cell whose firing is shown in Fig. 1A. The response, which was strong in this case, contained an excitatory component (indicated by a black area labelled $\bar{a}$ ), i.e., an elevation of the firing probability above the mean level to the left of the reference zero time ( $\tau=0 \mathrm{~ms}$ ), and a subsequent slight but longerlasting firing rate reduction (not visible at this magnification and short time scale). This reduction could be secondary to the preceding excitation and, hence, depend quantitatively on it. This was the case: Evaluating the firing rate reduction in the same way as the excitatory component by an integral $\bar{b}$ and correlating the two measures for 50 runs from 18 Renshaw cells yielded a strong linear correlation with a coefficient of 0.886 ( $P<0.001$, two-tailed). This finding may be explained by an afterhyperpolarization which grows in size and duration with the number of spikes in a burst. It is in keeping with the results of Walmsley and Tracey (1981) who in intracellular recordings from Renshaw cells saw afterhyperpolarizations after bursts of discharges, but hardly at all after single spikes (but see Hultborn and Pierrot-Deseilligny 1979), nor after recurrent EPSPs without spike discharge.

To analyse the dependency of the cell discharge on input pulses occurring before the reference pulses at $\tau=0 \mathrm{~ms}$ in the PSTH, "conditional" PSTHs (see Methods) were calculated. Part A of Fig. 2 shows an array of such PSTHs. These conditional PSTHs were calculated for varied $\delta$ 's, as indicated by the figures at the right. In the lower PSTH $(\delta=7.5 \mathrm{~ms})$, the response of the Renshaw cell to the conditioning 


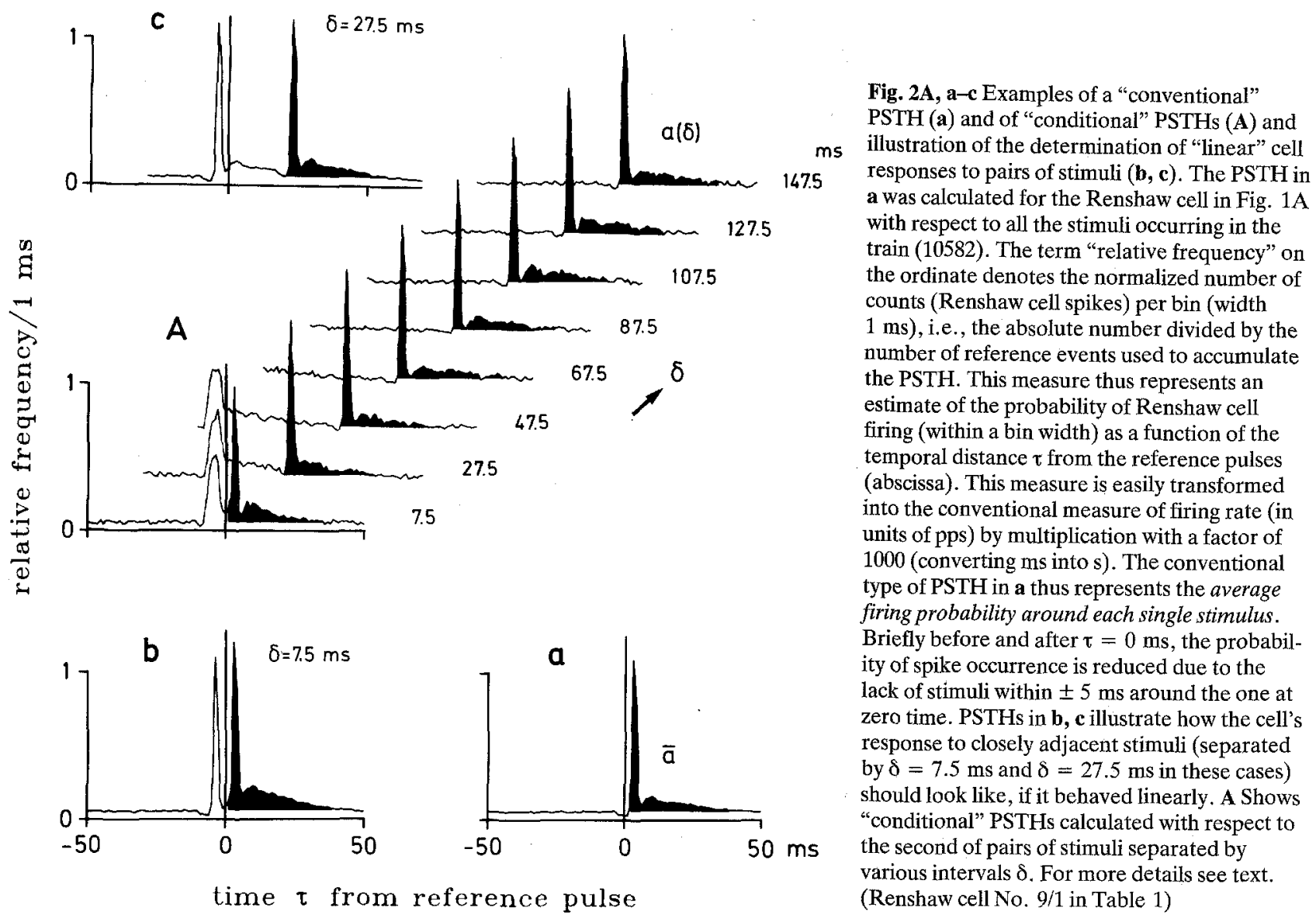

pulses is reflected in the peak to the left of the time origin ( $\tau=0 \mathrm{~ms}$ ), which is followed by its response to the test stimuli (at $\tau=0 \mathrm{~ms}$ ). The conditioning response is broader than the test response because the conditioning stimuli are dispersed over a window of $5 \mathrm{~ms}$ width. The other conditional PSTHs calculated for successively increasing $\delta$ 's are staggered upwards, aligned with respect to the conditioning responses. In other words, each PSTH is shifted to the right by another $20 \mathrm{~ms}$ (increase of $\delta$ ) with respect to the preceding PSTH.

The conditioning effects illustrated in Fig. 2A were quantified as follows. First consider the conventional PSTH calculated for all stimuli (Fig. 2a; lower right PSTH). After subtraction of the mean Renshaw cell discharge probability, the excitatory response to the right of the time origin was integrated within limits encompassing positive bin contents (here $38 \mathrm{~ms}$ ). This integral, visualized by the black area and designated $\bar{a}$, will henceforth be called "average Renshaw cell response". It represents the excess number of discharges contributed on average by each stimulus (thus being dimensionless). In this case, it was $\bar{a}=3.63$. Respective integrals labelled $a(\delta)$ were determined for the conditioned responses in Fig. 2A and were divided by the average Renshaw cell response, $\bar{a}$, to yield ratios. These were plotted as a function of $\delta$ (see the solid line in Fig. 3A). It is seen that the test responses are suppressed for quite a while after a conditioning stimulus.

In order to isolate the nonlinear component within this suppression, a linear model is needed as a reference. This model is based on the idea that, if the system under study behaved linearly, its test response (to a pulse at $\tau=0 \mathrm{~ms}$ ) would be equal to its average response superimposed on the same average response (to the conditioning pulse) but occurring $\delta \mathrm{ms}$ before (see Windhorst et al. 1983). Hence, the theoretical "linear response" to be compared with the actual conditioned response at $\delta=7.5 \mathrm{~ms}$ is calculated as follows (see Fig. 2b; lower left PSTH). Two average Renshaw cell responses as displayed in Fig. 2a (lower right PSTH) were superimposed at an interval of $\delta=7.5 \mathrm{~ms}$ as shown in Fig. 2b (lower left PSTH; after subtracting once the mean firing probability). This linear response should be compared with the actual conditioned response at $\delta=7.5 \mathrm{~ms}$ just above, i.e., the lowest conditional PSTH in Fig. 2A. 

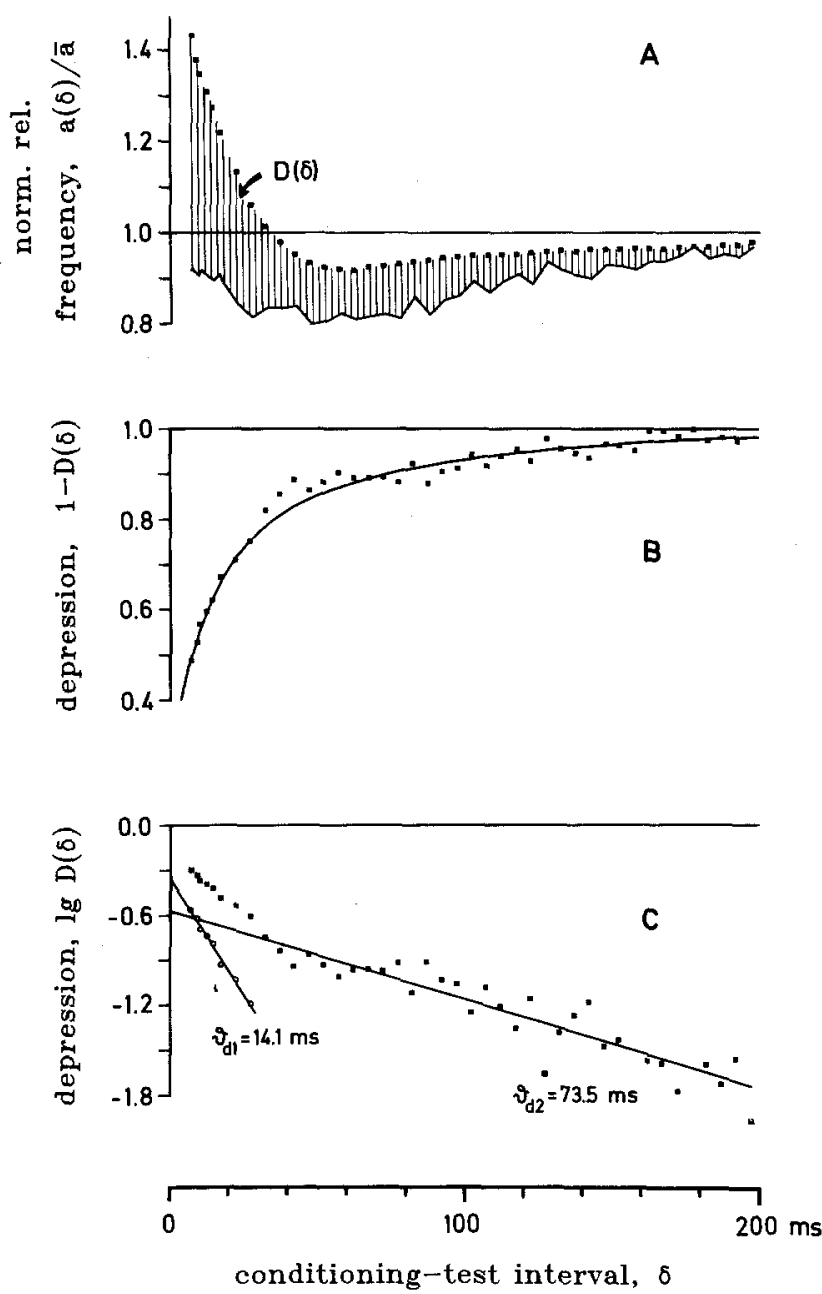

Fig. 3A-C. Time course of depression. Same cell as in Figs. 1A and 2. A Shows, as a function of $\delta$, the normalized Renshaw cell responses, $a(\delta) / \bar{a}$ (solid line), and their "linear" responses expected for linear behaviour (dots). The differences indicated by vertical hatching and labelled $D(\delta)$ are reproduced in $\mathbf{B}$, such that $\mathrm{D}(\delta)$ is plotted downwards as the deviation from $1(1-\mathrm{D}(\delta))$. C Is a semilogarithmic plot of $\mathrm{D}(\delta)$ (dots). A regression line (flat) was fitted to the right set of dots $(\delta \geq 32.5 \mathrm{~ms})$ and was used to calculate the long time constant, $\theta_{\mathrm{d} 2}$, and an ordinate intercept, $\mathrm{D}_{02}$. After subtraction of the values resulting from this latter exponential, the depression values for small $\delta$ 's $(\delta<32.5 \mathrm{~ms}$; open circles) were fitted by another regression line (steep), which yielded the small time constant, $\vartheta_{\mathrm{d} 1}$, and an ordinate intercept, $\mathrm{D}_{01}$. From these values we calculated the solid curve in $\mathbf{B}$, which fits the dots quite neatly

Another example for $\delta=27.5 \mathrm{~ms}$ is shown in Fig. $2 \mathrm{c}$ (upper left PSTH), corresponding to the second conditional PSTH from below in Fig. 2A. It is seen that, for these small $\delta$ 's, the second (test) response (black area) should indeed be larger rather than smaller than the average response, a, in Fig. 2a. In this way, linear responses were determined for varied $\delta$ 's, normalized (divided by $\bar{a}$ ) and plotted as dots in Fig. 3A. (For technical reasons, such linear responses could be determined only up to $\delta=$ $200 \mathrm{~ms}$, whereby the following analysis was restricted to this upper limit.) Note that for $\delta$ 's above about $30 \mathrm{~ms}$, these normalized linear responses are smaller than 1, which reflects the long-lasting, suppression of the average Renshaw cell response mentioned above. The difference between the dots and the solid line (hatched and labelled $\mathrm{D}(\delta)$ ) reflects the nonlinear component of depression. Hence, the effects of suppressed firing probability apparent in the average cell response and assigned to afterhyperpolarization are thereby eliminated from the subsequent analysis. $\mathrm{D}(\delta)$ will be taken to be positive, so that Fig. 3B shows $1-D(\delta)$. Such curves will henceforth simply be called "conditioning curves".

\section{Time constants of pure depression}

The curve in Fig. 3B reflects pure depression. This situation was analysed first. Generally, depression could be described as the sum of two exponentials:

$$
\mathrm{D}(\delta)=\mathrm{D}_{01} \cdot \exp \left(-\delta / \vartheta_{\mathrm{d} 1}\right)+\mathrm{D}_{02} \cdot \exp \left(-\delta / \vartheta_{\mathrm{d} 2}\right) \text {. }
$$

The constants in (1) were determined conventionally by plotting $\lg \mathrm{D}(\delta)$ against (linear) $\delta$ (Fig. $3 \mathrm{C}$ ). Using the least-squares method, a linear regression line (flat) was first fitted to the right set of solid dots starting from $\delta=32.5 \mathrm{~ms}$ and yielded a time constant of $\vartheta_{\mathrm{d} 2}=73.5 \mathrm{~ms}$ (see right label in C) and an ordinate value of $\lg \mathrm{D}_{02}=-0.57$ corresponding to $\mathrm{D}_{02}=0.27$. The deviation of the residual depression values at $\delta<32.5 \mathrm{~ms}$ from this regression line points to a component with a short time constant. To determine the constants of this component, the usual "peeling" procedure was employed. That is, the residual depression values and the ordinate values of the (flat) regression line at corresponding $\delta$ 's were expressed in linear terms (inverse logarithms). Their corresponding differences were determined and replotted in logarithmic ordinate values (open circles in Fig. 3C). Then another (steep) regression line was fitted to these points, yielding a time constant of $\vartheta_{\mathrm{d} 1}=14.1 \mathrm{~ms}$ (see left label in C) and a value of $\mathrm{D}_{01}=0.44$. These constants were used to calculate, as a control, a smooth fit to the dots in Fig. 3B according to (1) (solid curve). This fit is quite good.

Whereas in most cases the determination of time constants and ordinate intercepts by the above procedure (calculation of regression lines from semilogarithmic plots) proved satisfactory and yielded good fits to the conditioning curves, these fits were occasionally improved in linear coordinates by slightly changing the parameters appropriately. This was particularly helpful in fitting curves with mixed facilitation/depression. 
Table 1. Parameters of exponentials fitted to depression

\begin{tabular}{|c|c|c|c|c|c|}
\hline Cell number & $\begin{array}{l}\text { Mean stimulus } \\
\text { rate (pps) }\end{array}$ & $\mathrm{D}_{01}$ & $\mathrm{D}_{02}$ & $\begin{array}{l}\vartheta_{\mathrm{dl}} \\
(\mathrm{ms})\end{array}$ & $\begin{array}{l}\vartheta_{\mathrm{d} 2} \\
(\mathrm{~ms})\end{array}$ \\
\hline $6 / 6^{a}$ & 1 & 0.43 & 0.30 & 14.1 & 83.1 \\
\hline $6 / 6^{\mathrm{a}}$ & $\mathrm{m}$ & 0.26 & 0.25 & 13.0 & 75.0 \\
\hline $6 / 6^{\mathrm{a}}$ & $\mathrm{h}$ & 0.29 & 0.10 & 13.1 & 65.3 \\
\hline $7 / 1$ & l & 0.87 & 0.05 & 8.3 & 161.1 \\
\hline $7 / 2$ & 1 & 0.40 & 0.23 & 10.2 & 77.0 \\
\hline $7 / 2$ & $\mathrm{~m}$ & 0.57 & 0.16 & 11.1 & 75.3 \\
\hline $7 / 3$ & 1 & 0.53 & 0.11 & 17.5 & 94.4 \\
\hline $7 / 3$ & 11 & 0.76 & 0.10 & 15.5 & 109.3 \\
\hline $7 / 3$ & $\mathrm{~m}$ & 0.67 & 0.04 & 10.3 & 126.5 \\
\hline $7 / 3$ & 1 & 0.59 & 0.21 & 22.6 & 69.7 \\
\hline $7 / 3$ & $\mathrm{~m}$ & 0.30 & 0.17 & 16.6 & 68.0 \\
\hline $7 / 3^{\mathrm{R}}$ & $\mathrm{m}$ & 0.42 & 0.15 & 18.5 & 80.6 \\
\hline $7 / 3^{P}$ & 1 & 0.72 & 0.14 & 21.1 & 113.4 \\
\hline $7 / 3^{P}$ & $\mathrm{~m}$ & 0.18 & 0.23 & 25.2 & 50.3 \\
\hline $8 / 1$ & $\mathrm{~m}$ & 0.20 & 0.11 & 7.4 & 62.0 \\
\hline $9 / 1$ & 1 & 0.43 & 0.22 & 19.8 & 90.3 \\
\hline $9 / 1$ & 11 & 0.44 & 0.27 & 14.1 & 73.5 \\
\hline $9 / 1$ & $\mathrm{~m}$ & 0.52 & 0.18 & 7.5 & 63.1 \\
\hline $9 / 1$ & $\mathrm{~m}$ & 0.32 & 0.11 & 14.5 & 111.6 \\
\hline \multirow[t]{2}{*}{ mean $\pm S D$} & 1 & $0.59 \pm 0.17$ & $0.16 \pm 0.07$ & $14.9 \pm 5.4$ & $88.3 \pm 28.2$ \\
\hline & $\mathrm{m}$ & $0.38 \pm 0.17$ & & & \\
\hline
\end{tabular}

The second column indicates the mean stimulus rate used, where "l" stands for "low" (between 9.5 and 13 pps), "m" for "medium" (between 20 and $23 \mathrm{pps}$ ), and "h" for "high" (ca. $45 \mathrm{pps).} \mathrm{The} \mathrm{vertical} \mathrm{bars} \mathrm{right} \mathrm{to} \mathrm{some} \mathrm{l's,} \mathrm{m's,} \mathrm{and} \mathrm{h's} \mathrm{indicate} \mathrm{that} \mathrm{these} \mathrm{cells} \mathrm{were}$ tested twice or three times by stimulation of the same nerve or ventral root at the same strength, but at different mean rates. Two means and standard deviations (SDs) were calculated for the $\mathrm{D}_{01}$ because they differed significantly for low and medium mean stimulus rates

${ }^{a}$ This cell was tested at three different stimulus rates $(\mathrm{I}=11.4 \mathrm{pps}, \mathrm{m}=22.6 \mathrm{pps}$ and $\mathrm{h}=44.9 \mathrm{pps})$, which were obtained by replaying a tape with a pseudo-random pattern at three different speeds. This resulted in three minimal intervals in the stimulus patterns ( $1,11.4$ ms; $\mathrm{m}, 5.6 \mathrm{~ms} ; \mathrm{h}, 2.8 \mathrm{~ms}$ ). Therefore, the values in the first and third row were not taken into account in calculating the means and standard deviations. This cell is the only one in this collection of cells from a non-spinalized cat

${ }^{R}$ same stimulus parameters as in preceding line, i.e., repetition of the test

${ }^{\mathrm{P}}$ parallel activation of another nerve with an independent random pattern at about the same mean rate

\begin{abstract}
The excitatory Renshaw cell response in Fig. 2a consisted of two components, an early sharp peak followed by a smaller longer tail. It could be presumed that these two components were responsible for the short and long depression time constant, respectively. However, this was not the case. Firstly, there were average cell responses with essentially only one early peak (see, e.g., Fig. 6, left column), whose conditioning curves also had the two time constants. See also Fig. 5A, where a cell with a single early peak had a long depression time constant. Secondly, if conditioning curves were determined separately for the early and late response component, whereas the latter usually was depressed more strongly than the former, it also had a short and a long time constant (e.g., the cell illustrated in Figs. 1-3).
\end{abstract}

Values of time constants $\left(\vartheta_{\mathrm{di}}\right)$ and ordinate.intercepts $\left(D_{0 \mathrm{i}}\right)$ thus obtained from 19 experimental runs on 6 Renshaw cells (all tested with peripheral nerve stimulation except for the first cell which was activated by $\mathrm{L}_{7}$ ventral root stimulation) are collected in Table 1. In some cases, several values were obtained for the same cell tested at different mean rates (indicated by vertical bars in the second col- umn) and/or with different nerve inputs. Further data on the slow decay of depression from three more cells are assembled in Table 3 (see below).

\section{Time constants of pure facilitation}

Whereas pure depression was found relatively often, pure facilitation was rare in our material. This was due to the fact that facilitation appeared only under certain conditions, i.e., with small average Renshaw cell responses ( $\bar{a}$ ), which was obtained with weak input coupling and/or at medium or high mean stimulus rates (unpublished data).

An example of pure facilitation is illustrated in Fig. 4. The $\delta$-curve (solid line in part A) is noisier than that in Fig. 3A, in part due to the much smaller average Renshaw cell response ( $\overline{\mathrm{a}}=0.43$ ), which is apparent from the inset at the top. The procedure used to analyse the time course of facilitation was the 
same as that in Fig. 3. Based on the work of Mallart and Martin (1967) and Magleby (1973) on facilitation at frog neuromuscular junctions, the hypothesis was that two exponentials would fit this time course:

$$
F(\delta)=F_{01} \cdot \exp \left(-\delta / \vartheta_{\mathrm{f} 1}\right)+F_{02} \cdot \exp \left(-\delta / \vartheta_{\mathrm{f} 2}\right)
$$

where facilitation is defined equivalently to depression (1).

The constants determined for this and another case are collected in Table 2, where the second line contains the parameters of the exponentials fitted to the example in Fig. 4B. It is apparent that the values in Table 2 are comparable to those obtained for pure depression (Table 1).

\section{Reconstructed time course of mixed facilitation and depression}

In a number of cases, conditioning effects were composed of early facilitation followed by subsequent depression, as illustrated by the two examples in Fig. 5. These cases are not as easily analysed as the previous cases of pure facilitation or pure depression. From the preceding analysis, each facilitation and depression could have been represented by two exponentials. However, this would have given an unnecessarily complicated approach. Because early facilitation was so dominant in curves such as those illustrated in Fig. 5, these could be represented by two exponentials only: one representing the late depression (with a relatively long time constant) and one representing the early facilitation (with a briefer time constant). The approach taken was thus as follows:

$$
\begin{aligned}
G(\delta) & =1-D(\delta)+F(\delta) \\
& =1-D_{0} \cdot \exp \left(-\delta / \vartheta_{d}\right)+F_{0} \cdot \exp \left(-\delta / \vartheta_{f}\right) .
\end{aligned}
$$

The constants for four cases are collected in Table 3. Note that the time constants of depression, $\vartheta_{\mathrm{d}}$, are within the range of the long time constants, $\vartheta_{\mathrm{d} 2}$, found for pure depression, and that those of facilitation, $\vartheta_{\mathrm{f}}$, are well compatible with the $\vartheta_{\mathrm{f} 1}$ found for pure facilitation, although the 33 and $35 \mathrm{~ms}$ values are somewhat long.

\section{Relationships between some parameters of fitted exponentials}

The short time constants of depression, $\vartheta_{\mathrm{d} 1}$, were not significantly correlated with the related ordinate
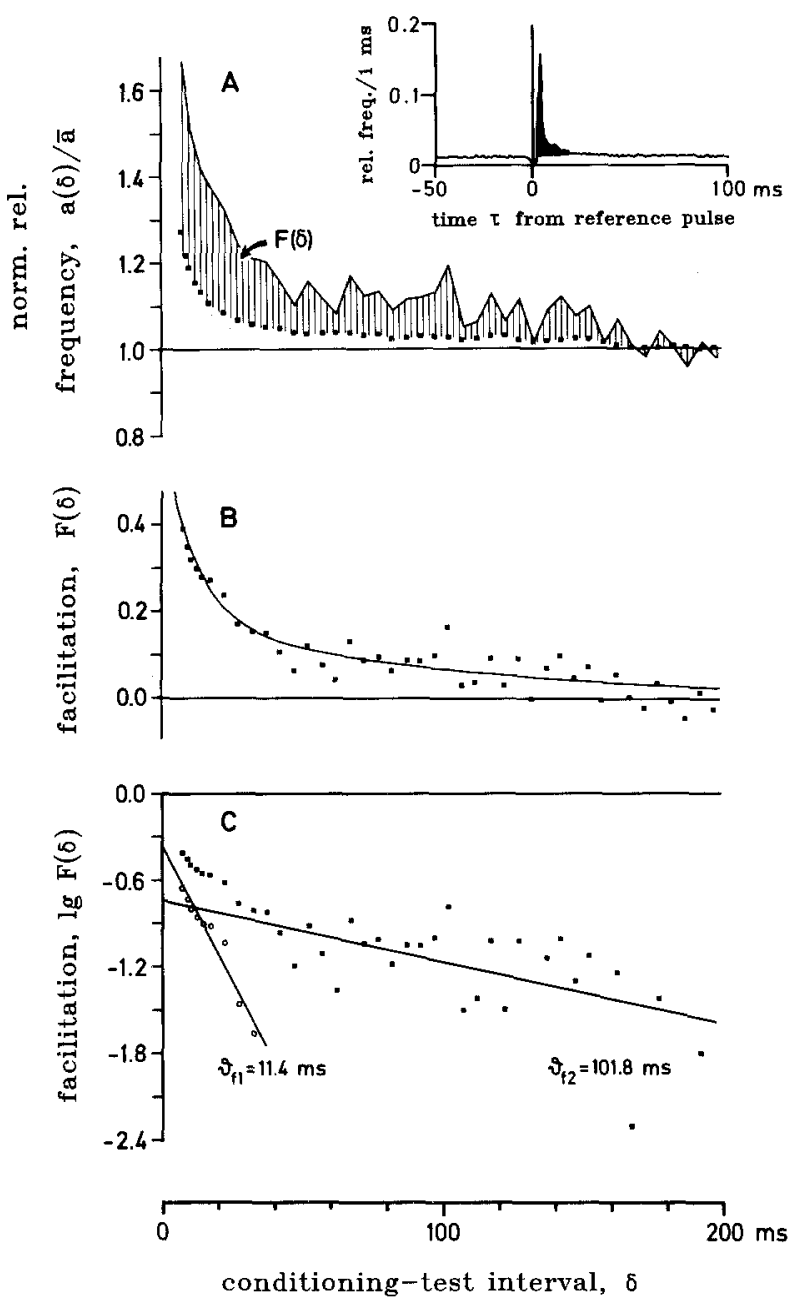

Fig. 4A-C. Time course of facilitation. This Renshaw cell was activated by random stimulation of the lateral gastrocnemiussoleus nerve at $230 \mathrm{mV}$ and a mean rate of $21.9 \mathrm{pps}$. The structure of this figure is basically the same as that of Fig. 3. (Cell No. 7/2 in Table 2). The inset on top shows the average Renshaw cell response to 15967 reference events

Table 2. Parameters of exponentials fitted to facilitation

\begin{tabular}{lllllr}
\hline Cell number & $\begin{array}{l}\text { Mean stimulus } \\
\text { rate }(\mathrm{pps})\end{array}$ & $\mathrm{F}_{01}$ & $\mathrm{~F}_{02}$ & $\begin{array}{l}\vartheta_{\mathrm{f} 1} \\
(\mathrm{~ms})\end{array}$ & \multicolumn{1}{l}{$\begin{array}{l}\vartheta_{\mathrm{f} 2} \\
(\mathrm{~ms})\end{array}$} \\
\hline $3 / 2$ & $\mathrm{~m}$ & 0.72 & 0.09 & 23.4 & 93.2 \\
$7 / 2$ & $\mathrm{~m}$ & 0.45 & 0.18 & 11.4 & 101.8 \\
mean & & 0.59 & 0.14 & 17.4 & 97.5 \\
\hline
\end{tabular}

intercepts, $\mathrm{D}_{01}$. By contrast, a negative linear correlation existed between the long time constants of depression, $\vartheta_{\mathrm{d} 2}$, and the related ordinate intercepts, $\mathrm{D}_{02}$, with a correlation coefficient of $-0.67(n=21 ; P$ $<0.001)$. 


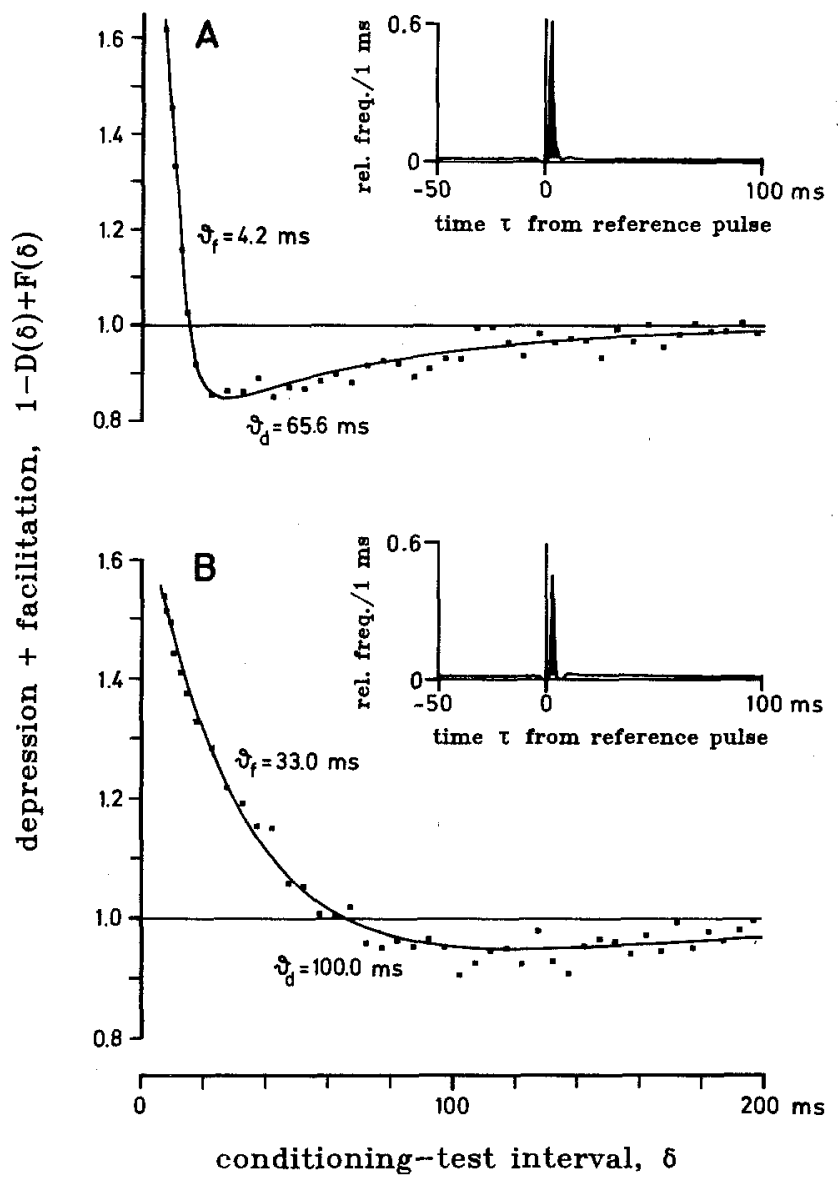

Fig. 5A-B. Two examples of "mixed" facilitation and depression (cell Nos. $1 / 1$ and $3 / 3$ in Table 3 ). The differences between the actual $\delta$-curve and the curve expected from linear summation are plotted as dots vs. $\delta$. The continuous lines were fitted to these dots by the sum of two exponentials, one representing depression and the other facilitation (see text). Mean stimulus rates: $\mathbf{A}, 12.0 \mathrm{pps}$; B, 20.2 pps. The two insets in $\mathbf{A}$ and $\mathbf{B}$ show the conventional PSTHs of the respective Renshaw cells to 5958 (A) and 18791 (B) reference events

There was no significant correlation between the long $\left(\vartheta_{\mathrm{d} 2}\right)$ and the short $\left(\vartheta_{\mathrm{d} 1}\right)$ time constant of depression. Also, these two time constants did not depend significantly on mean stimulus rate.

There was a negative correlation between $\mathrm{D}_{01}$ and
$\mathrm{D}_{02}(n=17)$ with a correlation coefficient of $-0.54(P$ $<0.02)$. This may be understood from the fact that depression values are limited to an upper boundary of 1 . So if $D_{01}$ is large, $D_{02}$ must be small, and vice versa. Note that this does not hold true for facilitation. On the average, the ordinate intercepts, $D_{01}$, related to the small time constant, are smaller at medium than at low mean stimulus rates (WilcoxonMann-Whitney test, two-sided, $P<0.05$ ). This is not so for $D_{02}$ (even including the values from Table 3 ), but it is again for the sum of $D_{01}+D_{02}$, which corresponds to the initial overall depression at $\delta=0 \mathrm{~ms}$. This is in keeping with results obtained on many more $\delta$-curves from more cells with a different method (i.e., integrating depression over the first $200 \mathrm{~ms}$ of $\delta$; Windhorst et al. unpublished).

\section{Comparison of homosynaptic with heterosynaptic conditioning}

To see whether the depressant conditioning effects described in the preceding sections were truly homosynaptic (i.e., confined to the pathway stimulated), we performed experimental runs in which two motor nerves or nerve branches were stimulated with independent random patterns of similar mean rate. Figure 6 shows characteristic results from a Renshaw cell excited by the medial gastrocnemius (MG) and the lateral gastrocnemius-soleus (LGS) nerves at medium rates. The respective average responses as assessed by PSTHs (A and E) were slightly different. "Auto-conditioning" (conditioning and test stimuli in the same train) exhibited depression (lower rows: C, $\mathrm{D}$ and $\mathrm{G}, \mathrm{H}$ ). "Cross-conditioning" (conditioning stimuli from the train parallel to the one yielding the reference or test events) yielded facilitation of different strength at small intervals, $\delta$, which was almost certainly due to spatial summation (B and F). At larger intervals, the reduction in Renshaw cell responses is largely accounted for by the "linear" responses (dots). That is, activation of a parallel (heterosynaptic) input does not induce a significant

Table 3. Parameters of exponentials fitted to mixed facilitation/depression

\begin{tabular}{|c|c|c|c|c|c|}
\hline Cell number & $\begin{array}{l}\text { Mean stimulus } \\
\text { rate (pps) }\end{array}$ & $F_{01}$ & $D_{02}$ & $\begin{array}{l}\vartheta_{\mathrm{f} 1} \\
(\mathrm{~ms})\end{array}$ & $\begin{array}{l}\vartheta_{\mathrm{d} 2} \\
(\mathrm{~ms})\end{array}$ \\
\hline $1 / 1$ & 11 & 6.81 & 0.23 & 4.2 & 65.6 \\
\hline $1 / 1$ & $\mathrm{~m}$ & 3.00 & 0.28 & 8.2 & 70.0 \\
\hline $3 / 3$ & $\mathrm{~m}$ & 0.95 & 0.25 & 33.0 & 100.0 \\
\hline $3 / 4$ & $\mathrm{~m}$ & 0.80 & 0.40 & 35.0 & 80.0 \\
\hline mean $\pm S D$ & & $2.89 \pm 2.80$ & $0.29 \pm 0.08$ & $20.1 \pm 16.15$ & $78.9 \pm 15.30$ \\
\hline
\end{tabular}



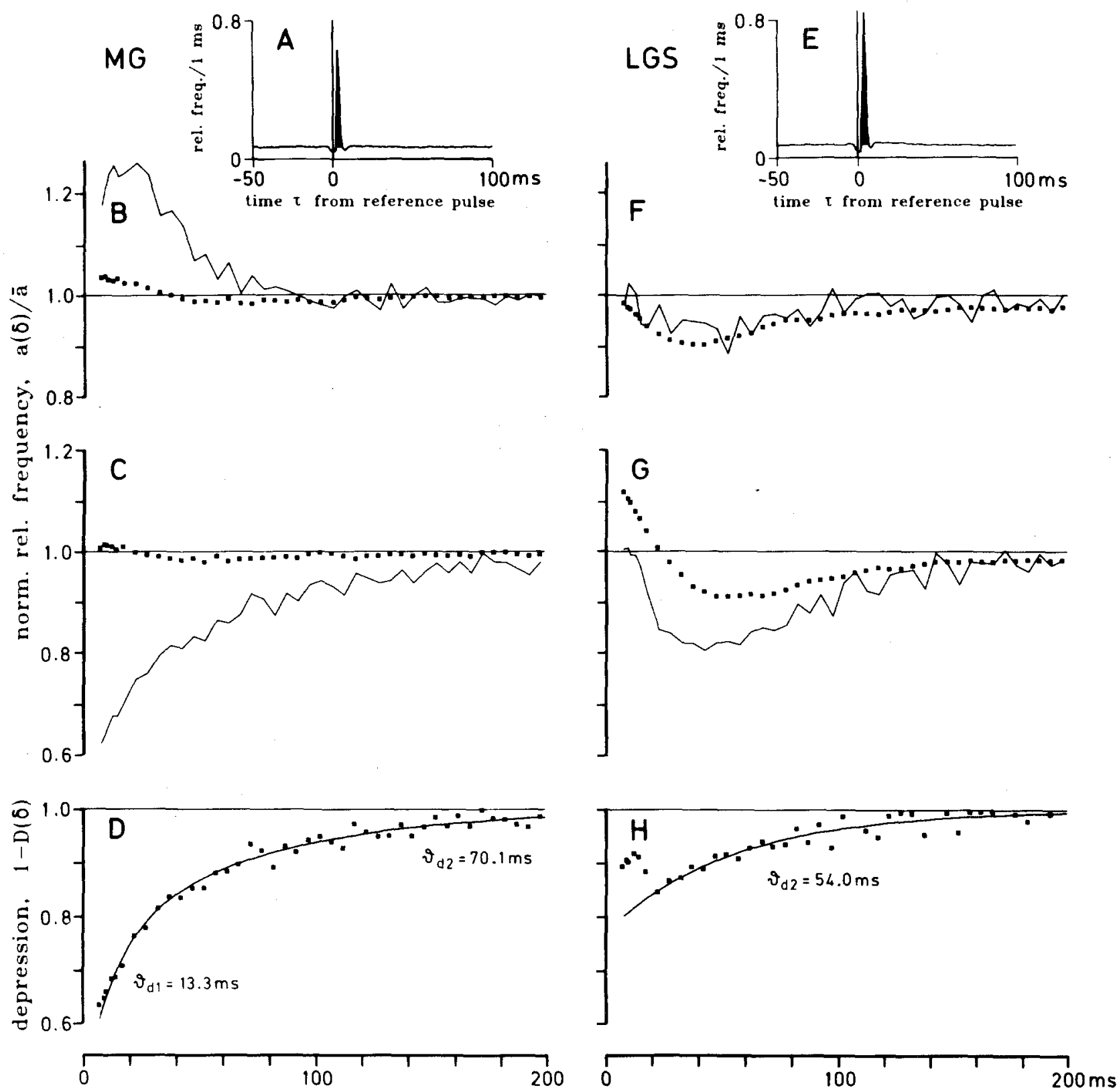

conditioning-test interval, $\delta$

Fig. 6A-H. Comparison of homo- and heterosynaptic conditioning effects. The medial gastrocnemius (MG) and lateral gastrocnemiussoleus (LGS) nerves were stimulated (with $400 \mathrm{mV}$ each) in parallel with independent random patterns at mean rates of 22.7 and $22.3 \mathrm{pps}$, respectively. Average Renshaw cell discharge rate: $76.8 \mathrm{pps}$. Each column refers to Renshaw cell responses which were computed with respect to a particular stimulus train (yielding the reference events) as indicated by "MG" or "LGS" on top. The average Renshaw cell responses are shown by PSTHs on top (number of reference events: $\mathbf{A}, 20880 ; \mathbf{E}, 20489$ ). The second row (B and F) displays the conditioning effects exerted by the "parallel" (heterosynaptic) input (actual responses: solid line; "linear" values: dots). These effects were computed in a similar way to those of the "own" (homosynaptic) input by selecting the conditioning stimuli from the parallel stimulus train. The "linear" values were also computed as in the homosynaptic case, but with the average response to the parallel input as conditioning response. The third row ( $\mathbf{C}$ and $\mathbf{G}$ ) shows the homosynaptic conditioning effects (actual and "linear" values). The fourth row (D and $\mathbf{H})$ exhibits the differences between the actual and linear values in the third row and curves fitted with the following values: $\mathbf{D}: \mathrm{D}_{01}=0.29$, $\vartheta_{\mathrm{d} 1}=13.3 \mathrm{~ms}, \mathrm{D}_{02}=0.27, \vartheta_{\mathrm{d} 2}=70.1 \mathrm{~ms}(\sec (1)) ; \mathbf{H}: \mathrm{D}_{02}=0.24, \vartheta_{\mathrm{d} 2}=54.0 \mathrm{~ms}$ 
depression other than that resulting from afterhyperpolarization. Yet homosynaptic depression is prominent. Interestingly, the early auto-conditioning curve for the lateral gastrocnemius-soleus nerve (Fig. . 6H) shows an early facilitation component in statu nascendi, which has not been fitted because of the scatter of the points. Although the parameters of the exponentials fitted to the points in Fig. $6 \mathrm{D}$ and $\mathrm{H}$ are well compatible with those listed in Table 1, they have not been included in this table because they were obtained under a different experimental condition (activation of parallel input channels).

\section{Discussion}

The present study was designed to quantitate Renshaw cell behaviour in response to fluctuations of motoneurone firing around mean rates corresponding to those of natural maintained discharge. The random stimulus patterns used are optimal for this purpose. Yet using Renshaw cell firing as an output variable certainly poses some problems of interpretation of the conditioning effects obtained, not all of which can be resolved with the present approach.

Renshaw cell responses to test pulses are conditioned by preceding pulses in the random sequence of stimuli. Three main patterns have been observed: pure facilitation, pure depression and mixed early facilitation and late depression. Our approach to quantitatively describe the time course of the first two by the sum of two exponentials was based on a similar approach taken by Mallart and Martin (1967) and Magleby (1973) to describe facilitation at the frog neuromuscular junction. This assumption was validated by our data.

\section{Possible causes of conditioning effects}

The conditioning effects described in Results may have two main sources: pre- and postsynaptic ones.

The long-facilitation $\left(\vartheta_{f 2}\right)$ is very probably due to a presynaptic mechanism. From studies on frog and mammalian neuromuscular junctions, the process called "facilitation" can be described by two time constants of the order of $35-50 \mathrm{~ms}$ and $250-300 \mathrm{~ms}$ (Mallart and Martin 1967; Magleby 1973). These two time courses suggest different underlying mechanisms. For example, Mallart and Martin (1967) considered the possibility that the fast facilitation could reflect an increased probability (p) of release of available transmitter, whereas the slow process could reflect augmented mobilization of transmitter for release, i.e., an increase in the number (n) of quanta available for release. But other possibilities have also been discussed (see, e.g., Magleby 1973). In any case, the occurrence of two time constants of facilitation at the neuromuscular junction suggests that a similar duality also occur at the cholinergic recurrent collateral synapses on Renshaw cells. However, there are two complications. Firstly, as compared to the time constants at the frog neuromuscular junction, those found in Renshaw cell facilitation $\left(\vartheta_{\mathrm{f} 1}\right.$, Table 2, second row, Table 3, first two rows) are relatively small. This may be related to the different temperatures at which the different investigations were performed, since at least the fast time constant at the endplate is very sensitive to temperature changes. Balnave and Gage (1970), apparently only observing the short time constant at the frog endplate, found that it decreased exponentially with increasing temperature, from ca. $120 \mathrm{~ms}$ at $12.3^{\circ} \mathrm{C}$ to ca. $15 \mathrm{~ms}$ at $28^{\circ} \mathrm{C}$, with a $\mathrm{Q}_{10}$ of 3.9. Extrapolating this relationship (their Fig. 2) to $35^{\circ} \mathrm{C}$ would yield time constants of ca. $5 \mathrm{~ms}$. Whether or not this extrapolation is allowed, our short time constants $\left(\vartheta_{\mathrm{f} 1}\right)$ would appear to lie in a reasonable range if it is assumed that they reflect synaptic facilitation. This might then also apply to the longer time constants $\left(\vartheta_{\mathrm{f} 2}\right)$. Whilst this reasoning might make a presynaptic mechanism for the short time constant plausible, it does not exclude other mechanisms, since a second source of early facilitation may be the temporal summation of EPSPs, which in Renshaw cells appear to be of long duration (lasting up to $60 \mathrm{~ms}$ or so; see Eccles et al. 1961; Walmsley and Tracey 1981). This summation might also give rise to a facilitation with time constants of the order of $15 \mathrm{~ms}$. Thus, this and truly synaptic facilitation probably add to the overall effect.

The long depression $\left(\vartheta_{\mathrm{d} 2}\right)$ is also most likely to be due to a presynaptic origin, for the following reasons.

With regard to the long depression, it is unlikely that the summation of afterhyperpolarization contributes very much because of the reasons given in Results, that is, because we have essentially excluded such a contribution by subtracting the "linear response". This argument is supported by results of Eccles et al. (1961) who in passing recorded intracellularly from a Renshaw cell and found the second of a pair of EPSPs (elicited by ventral root stimulation) suppressed for more than $150 \mathrm{~ms}$. We could fit their data points (their Fig. 6J) with two exponentials (with time constants of the order of 30 and $100 \mathrm{~ms}$ ), although not well so because of the scatter of their data. A contribution to depression could also come from mutual inhibition between Renshaw cells (Ryall 1970,1981 ). But if so, it is probably small because activation of a synergistic parallel input (Fig. 6) does not produce considerable depression above that 
accounted for by afterhyperpolarization. This is in keeping with the expectation that mutual inhibition be strongest between Renshaw cells getting their respective excitatory inputs from antagonistic $\alpha$ motoneurones (Ryall 1981), whereas it should be relatively weak among Renshaw cells receiving their main input from homonymous motoneurones. Yet late (and strong) depression also occurs with activation of purely homonymous motor axons (Figs. 3 and 6).

Synaptic depression was also found at frog and mammalian neuromuscular junctions (e.g., Thies 1965; Betz 1970; Christensen and Martin 1970), at the Ia-motoneurone synapse (Curtis and Eccles 1960; Kuno 1964; Čapek and Esplin 1977; Collins III et al. 1984) as well as at hippocampal synapses (e.g., McNaughton 1980). It might result from transmitter depletion (Thies 1965; Capek and Esplin 1977), but this is probably not the only factor contributing (Betz 1970; for review see Silinski 1985).

Capek and Esplin (1977) studied depression at the cat Ia-motoneurone synapse with short trains of regularly spaced pulses and interpreted the results in terms of a simple mathematical model of presynaptic transmitter depletion. They found that the rate of replenishment ( $r$ ) of transmitter stores increased with an increase in the rate of activation. At $10 \mathrm{pps}$, the replenishment rate attained 3.1-4.1/s (corresponding to a time constant of 0.32 to $0.24 \mathrm{~s}$ ). These time constants are greater than ours for Renshaw cell depression (of the order of 80-90 ms; Tables 1 and 3 ). This seeming discrepancy could result from the different stimulus patterns. The pseudo-random patterns we used contained a large variety of intervals, in which smaller-than-average intervals abounded (exponential interval distribution!). That is, the physiologically "effective" rate in our experimental approach may have been higher than that expressed by the mean rate.

The origin of the short time constant of depression is not yet settled. Its near-identity to that of facilitation (as well as the correspondence of the respective long time constants: Tables 1-3) suggests that the mechanism underlying each time constant may be facilitated or depressed, depending upon various circumstances (see below). If this holds true and if the short and long components of facilitation are related to the probability $(\mathrm{p})$ of quantal release and the number (n) of releasable quanta, respectively (see above), depression should also affect both quantities. Indeed Betz (1970) argued that, at the frog neuromuscular junction, depression reduced both $p$ and $n$ (see also Silinski 1985). But whether these two effects have different time courses has still to be demonstrated. Capek and Esplin (1977) could not assess the short depression time constant because of their limitation of stimulus rate to $10 \mathrm{pps}$. Collins III et al. (1984), using short bursts of high-rate (167 pps) stimuli to single Ia fibres to study EPSP modification in homonymous $\alpha$-motoneurones, found depression at some connections (see below) that is suggestive of a short time constant (but no quantification of time course was attempted).

The balance between facilitation and depression is complicated and depends on a number of factors. For instance, McNaughton (1980) noted that raising the mean stimulus rate promoted facilitation and reduced depression. This is in accord with our results. An interesting new finding for Ia-motoneurone synapses is that the prevalence of facilitation or depression during high-frequency (167 pps) repetitive stimulation is associated with the strength of the particular (single-fibre) connection: Large EPSPs tend to depress, small ones to facilitate, and this occurs irrespective of motoneurone type (Collins III et al. 1984). Whether a similar trend exists at motor axon-Renshaw cell synapses has yet to be investigated.

In summary, the conditioning effects described in this paper probably result to a considerable extent from presynaptic processes of facilitation and depression which are also known from other synapses. However, the main open question is to what extent the nonlinear spike triggering mechanism in the Renshaw cell contributes to the conditioning effects described. The transformation of membrane potential fluctuations into spike sequences can only be studied by means of intracellular recording, which is very difficult, however.

\section{Physiological significance}

The present results are pertinent to possible mechanisms of physiological tremor. The power spectrum of this form of tremor has a local peak in the frequency range $8-12 \mathrm{~Hz}$, which probably results from unfused contractions of newly recruited motor units that discharge relatively regularly at such low rates (Allum et al. 1978; Christakos 1982) in a nearasynchronous way. In this case, the intervals between discharges of each of these motor units would be about 80 to $120 \mathrm{~ms}$ long, well within the range of depressant effects. There is evidence that single motoneurones recurrently inhibit themselves (van Keulen 1981). If the motoneurone fires rather regularly, the Renshaw cell response should be about the same to each motoneurone firing. However, motor units sometimes discharge doublets with short intervals followed by longer-than-average intervals, and successive intervals may be correlated negatively 
(Andreassen and Rosenfalck 1980). Under certain conditions, this alternation of short and long intervals may be accentuated (Bawa and Calancie 1983; Elble and Randall 1976). Andreassen and Rosenfalck (1980) adduced three possible mechanisms for such patterns: summation of motoneuronal afterhyperpolarization, recurrent inhibition and proprioceptive feedback. Also, Elble and Randall (1976) attributed the alternation of short and long intervals to recurrent inhibition. However, the Renshaw cell response to a motoneurone doublet would consist of two successive bursts. For a strong motor axon-Renshaw cell connection, which may be required for an efficient self-inhibition, the second response would be depressed. It is therefore not clear why this smaller response should have a significant influence on the length of the longer-than-average interval following the doublet. If Renshaw cell discharge does at all co-determine motoneurone interspike intervals, 10 then this depression of the second response to a doublet should rather reduce the second interval. Since, in comparison to regular motoneurone firing, a sequence of alternating short and long intervals increases the tremor amplitude, the described Renshaw cell nonlinearity would therefore be expected to suppress rather than enhance tremor. The actual sequence of short and long intervals is probably generated mainly by the summation of afterhyperpolarization in motoneurones.

Acknowledgements. This work was partly supported by the Deutsche Forschungsgemeinschaft, SFB 33 ("Nervensystem und biologische Information") Göttingen. We are grateful to Mrs. B. Müller-Fechner and Mrs. U. deBuhr for technical assistance.

\section{References}

Allum JHJ, Dietz V, Freund HJ (1978) Neuronal mechanisms underlying physiological tremor. J Neurophysiol 41:557-57

Andreassen S, Rosenfalck A (1980) Regulation of the firing pattern of single motor units. J Neurol Neurosurg Psychiat 43: 897-906

Balnave RJ, Gage PW (1970) Temperature sensitivity of the time course of facilitation of transmitter release. Brain Res 21 297-300

Bawa P, Calancie B (1983) Repetitive doublets in human carpi radialis muscle. J Physiol (Lond) 339: 123-132

Betz WJ (1970) Depression of transmitter release at the neuromes cular junction of the frog. J Physiol (Lond) 206: 629-644

Čapek R, Esplin B (1977) Homosynaptic depression and transmitter turnover in spinal monosynaptic pathway. J Neurophysiol 40: 95-105

Christakos CN (1982) A study of the muscle waveform using a population stochastic model of skeletal muscle. Biol Cybern 44: $91-106$

Christakos CN, Windhorst U, Rissing R, Meyer-Lohmann J (1987) Frequency response of spinal Renshaw cells activated by stochastic motor axon stimulation. Neuroscience 23 : 613-623
Christensen BN, . Martin AR (1970) Estimates of probability of transmitter release at the mammalian neuromuscular junction. J Physiol (Lond) 210: 933-945

Collins WF III, Honig MG, Mendell LM (1984) Heterogeneity of group Ia synapses on homonymous $\alpha$-motoneurons as revealed by high-frequency stimulation of Ia afferent fibers. J Neurophysiol 52: 980-993

Curtis DR, Eccles JC (1960) Synaptic action during and after repetitive stimulation. J Physiol (Lond) 150: 374-398

Eccles JC, Eccles RM, Iggo A, Lundberg A (1961) Electrophysiological investigations on Renshaw cells. J Physiol (Lond) 159: 461-478

Elble RJ, Randall JE (1976) Motor-unit activity responsible for 8- to $12-\mathrm{Hz}$ component of human physiological finger tremor. J Neurophysiol 39: 370-383

Waase J (1963) Die Transformation des Entladungsmusters der Renshaw-Zellen bei tetanischer antidromer Reizung. Pflügers Arch ges Physiol 276: 471-480

GHamm TM, Sasaki S, Stuart DG, Windhorst U, Yuan C-S (1987) Distribution of single-axon recurrent inhibitory post-synaptic potentials in a single spinal motor nucleus in the cat. J Physiol (Lond) 388: 653-664

Hultborn H, Pierrot-Deseilligny E (1979) Input-output relations in the pathway of recurrent inhibition to motoneurones in the cat. J Physiol (Lond) 297: 267-287

Kuno M (1964) Mechanism of facilitation and depression of the excitatory synaptic potential in spinal motoneurones. J Physiol (Lond) 174: 100-112

Magleby KL (1973) The effect of repetitive stimulation on facilitation of transmitter release at the frog neuromuscular junction. J Physiol (Lond) 234: 327-352

Mallart A, Martin AR (1967) An analysis of facilitation of transmitter release at the neuromuscular junction of the frog. J Physiol (Lond) 193: 679-694

McNaughton BL (1980) Evidence for two physiologically distinct perforant pathways to the fascia dentata. Brain Res 199: 1-19

Ross H-G, Cleveland S, Haase J (1975) Contribution of single motoneurons to Renshaw cell activity. Neurosci Lett 1: 105-108

Ryall RW (1970) Renshaw cell mediated inhibition of Renshaw cells: patterns of excitation and inhibition from impulses in motor-axon collaterals. J Neurophysiol 33: 257-270

Ryall RW (1981) Patterns of recurrent excitation and mutual inhibition of cat Renshaw cells. J Physiol (Lond) 316: 439-452

Silinski EM (1985) The biophysical pharmacology of calciumdependent acetylcholine secretion. Pharmacol Rev 37: 81-132

Thies RE (1965) Neuromuscular depression and the apparent depletion of transmitter in mammalian muscle. J Neurophysiol 28: 423-442

a an Keulen L (1981) Autogenetic recurrent inhibition of individual spinal motoneurones of the cat. Neurosci Lett 21: 297-300

Walmsley B, Tracey DJ (1981) An intracellular study of Renshaw cells. Brain Res 223: 170-175

Windhorst U (1988) How brain-like is the spinal cord? Interacting cell assemblies in the nervous system. Springer, Berlin Heidelberg New York Tokyo

Windhorst U, Niemann U, Koehler W (1983) Analsyis of nonlinear physiological systems with single or multiple spike inputs and analogue or spike outputs. Biol Cybern 48: $159-163$

Windhorst U, Rissing R, Meyer-Lohmann J (1987) Aftereffects of stochastic synaptic Renshaw cell excitation on their discharge probability. Brain Res 408: 289-294

Received July 16, 1987 / Accepted March 28, 1988 